\title{
NUEVAS ESPECIES DE PALINOMORFOS DE LAS FORMACIONES SAN JOSÉ Y CHIQUIMIL (MIOCENO MEDIO Y SUPERIOR), NOROESTE DE ARGENTINA
}

\author{
LILIA RENÉ MAUTINO \\ Facultad de Ciencias Exactas, Naturales y Agrimensura, Universidad Nacional del Nordeste y Centro de Ecología Aplicada \\ del Litoral, Ruta 5 km 2,5 3400 Corrientes, Argentina. liliamautino @ yahoo.com.ar
}

\begin{abstract}
NEW PALYNOLOGICAL SPECIES FROM THE SAN JOSE AND CHIQUIMIL FORMATIONS (MIDDLE AND UPPER MIOCENE), NORTHWESTERN OF ARGENTINA. In this contribution are described taxonomically six new species of pollen of magnoliophytes from the San José and Chiquimil formations (middle and upper Miocene), Northwest of Argentina, as follow: Spinitricolpites anzoteguii sp. nov. and Periporopollenites vivianae sp. nov. (both Cactaceae), Retitetracolpites columelae sp. nov. (Nyctaginaceae), Areolipollis pirenii sp. nov. and A. insularis sp. nov. (both Acanthaceae) and Chenopodipollis minima sp. nov. (Amaranthaceae). The modern genus Trichocereus (A. Berger) Riccob., Echinopsis Zucc. and Opuntia Mill. (Cactaceae), Boungainvillea Comm. ex Juss. (Nyctaginaceae) and Justicia L. (Acanthaceae) are mentioned for the first time for the Paleocene-Pliocene interval in Argentina. The new species are included in paleocommunities that they would have integrated.
\end{abstract}

Key words: palynology, new species, Miocene, botanical affinities.

RESUMO - Nesta contribuição são descritas, taxonomicamente, seis espécies novas de grãos de pólen de magnoliófitas provenientes das formações San José e Chiquimil (Mioceno médio e superior), Noroeste da Argentina, como segue: Spinitricolpites anzoteguii sp. nov. e Periporopollenites vivianae sp. nov. (ambas Cactaceae), Retitetracolpites columelae sp. nov. (Nyctaginaceae), Areolipollis pirenii sp. nov. e A. insularis sp. nov. (ambas Acanthaceae) e Chenopodipollis minima sp. nov. (Amaranthaceae). Os gêneros atuais Trichocereus (A. Berger) Riccob., Echinopsis Zucc. y Opuntia Mill. (Cactaceae), Boungainvillea Comm. ex Juss. (Nyctaginaceae) e Justicia L. (Acanthaceae) são citados pela primera vez para para o intervalo Paleoceno-Plioceno na Argentina. As novas espécies são incluidas nas paleocomunidades vegetais das quais elas teriam participado.

Palabras-chave: palinologia, especies novas, Mioceno, afinidades botânicas.

\section{INTRODUCCIÓN}

El valle de Santa María o Yokavil es una depresión tectónica que corre de norte a sur y se encuentra inmerso en la unidad estructural de las Sierras Pampeanas. Abarca, al noroeste de Argentina, parte de las provincias de Catamarca, Tucumán y Salta. Está limitado por dos bloques de basamento de gran magnitud, al oeste la sierra de Quilmes o del Cajón y al este, la sierra del Aconquija y las Cumbres Calchaquíes (Figura 1). Las formaciones San José y Chiquimil se ubican en la secuencia basal del Grupo Santa María, y están intercaladas por la Formación Las Arcas. El contenido paleontológico de estas unidades mencionadas es variado, además de palinomorfos, se han hallado pelecípodos, gasterópodos, ostrácodos, foraminíferos, mamíferos, aves, peces, leños, hojas y frutos (Anzótegui, 2006; Barreda et al. 2007; Cione \& Baéz, 2007; Martínez, 2010; Morton, 2004).

La palinoflora de las formaciones San José y Chiquimil cuenta con 199 palinomorfos, de ellas 126 especies son magnoliófitas, 16 pinófitas, 37 pteridófitas, diez clorófitas y el mismo número de briófitas. El objetivo del trabajo es dar a conocer seis especies nuevas y sus afines actuales de: Cactaceae, Nyctaginaceae, Acanthaceae y Amaranthaceae. La mayoría de los taxones actuales afines a estas especies fósiles representan novedad en estratos del PaleocenoPlioceno argentinos; al considerar los requerimientos de hábito y hábitat de los mismos se corrobora la existencia de las paleocomunidades vegetales que fueran reconocidas mediante la palinoflora (Mautino, 2010).

Aportes palinológicos previos relacionados a ambas formaciones son los de Mautino \& Anzótegui (1998, 2000, 2002a,b), Mautino et al. (2004) y Mautino (2007), entre los que se hallan otros taxones nuevos: el género Malvapantocolporites Mautino, Cuadrado \& Anzótegui (Malvaceae) y nueve especies: Sphaeroplea miocenica Mautino (Sphaeropleaceae); Muricingulisporis verrucosus Mautino \& Anzótegui (Pteridaceae); Dictyophyllidites chiquimilense Mautino \& Anzótegui (Matoniaceae o Cyatheaceae); Malvacipolloides tucumanensis Mautino, Cuadrado \& Anzótegui; Malvapantocolporites rafaelii Mautino, Cuadrado \& Anzótegui; Malvapantocolporites sanjosesii Mautino, Cuadrado \& Anzótegui; Malvapantocolporites silvinites 
Mautino, Cuadrado \&Anzótegui; Echiperiporites santamariana Mautino, Cuadrado \& Anzótegui (todas Malvaceae); y Sparganiaceaepollenites delicata Mautino \& Anzótegui (Sparganiaceae-Typhaceae). Además se cuentan con dos combinaciones: Tubulifloridites minutus (Regali, Uesugui \& Santos) Mautino \& Anzótegui; Tubulifloridites spinosus (Van der Hammen ex Germeraad, Hopping \& Muller) Mautino \& Anzótegui y tres enmiendas: Lecaniella korsoddensis Batten, Koppelhus \& Nielsen emend. Mautino, Rhoipites alveolatus (Couper) Pocknall \& Crosbie emend. Mautino \& Anzótegui; Rhoipites baculatus Archangelsky emend. Mautino \& Anzótegui.

\section{MARCO GEOLÓGICO}

El Grupo Santa María es un complejo sedimentario que aflora más o menos paralelo al río Santa María sobre su margen derecha. Está integrado por seis formaciones (desde abajo): San José, Las Arcas, Chiquimil, Andalhuala, Corral Quemado y Yayasmayo (Bossi \& Palma, 1982). Basados en la fauna de mamíferos y dataciones radimétricas, Bossi \& Palma (1982) asignan al Grupo una edad Mioceno-Plioceno.

La base y techo de la Formación San José se encuentran en suave discordancia angular con la Formación Saladillo y con la Formación Las Arcas, respectivamente. Su espesor, en general, varía entre 250 y $315 \mathrm{~m}$ y hacia el norte alcanza los 1000 m, a la altura de la Quebrada de Agua Negra (Bossi \& Palma, 1982). Sedimentológicamente esta formación está conformada por areniscas finas a muy finas, en las que predomina el color amarillo-verdoso con estratificación de tipo lenticular, tabular, cruzada y/o laminación ondulítica. Estas areniscas están acompañadas por pelitas, de colores verde y castaño claro, en general macizas o con laminación paralela, pocas veces ondulíticas. Entre ellas se encuentran intercaladas capas de yeso, venas de micas y calizas biomicríticas.

Por otro lado, la Formación Chiquimil se encuentra por encima de Formación Las Arcas, y debajo de Andalhuala. El espesor de la formación varía entre $480 \mathrm{~m}$ en la localidad Entre Ríos, alcanzando un máximo de 1199 m en la Quebrada de Agua Negra (Ibáñez, 2001). La litología se caracteriza por la predominancia de colores amarillos claros, moderadamente salina y con venillas de yeso. Las areniscas finas se disponen en conjuntos gruesos (macizos, laminados paralelos o con estratificación cruzada) y alternan con limolitas y arcilitas de colores variados. En muchos niveles, en general los superiores, se intercalan conglomerados finos con rodados redondeados de vulcanitas (basaltos, andesitas, dacitas y metamórficos).

Las edades atribuídas a ambas formaciones son Mioceno medio para Formación San José y Mioceno superior para Formación Chiquimil obtenidas a partir mediciones radimétricas en tobas, de datos estratigráficos y paleontológicos (Bossi et al., 1998; Kleinert \& Strecker, 2001; Latorre et al., 1997).

\section{MATERIAL Y MÉTODOS}

Los palinomorfos de este trabajo provienen de muestras obtenidas de los perfiles realizados en cuatro localidades; dos de estos perfiles se realizaron en la Formación San José en las localidades Río Salinas y km 107 (Tucumán), y los restantes en las localidades Río Vallecito y Quebrada de Jujuil en la Formación Chiquimil (Catamarca) (Figura 1).

En las localidades Río Salinas y km 107 (mojón de la ruta Provincial $n^{\circ} 307$, próxima al Observatorio Astronómico de Ampimpa) ambas en la Provincia de Tucumán, los perfiles tuvieron un espesor de $70 \mathrm{~m}$ aproximadamente; de la localidad km 107 se obtuvieron 19 muestras fértiles y de la localidad Río Salinas, nueve. Por otro lado, el perfil de la localidad Río Vallecito tuvo una potencia de $350 \mathrm{~m}$ y las muestras fértiles fueron 24; en la localidad Quebrada de Jujuil (687 m perfilados), sólo 19 muestras arrojaron resultado positivo.

Las muestras han sido procesadas siguiendo el método convencional de eliminación de silicatos con ácido fluorhídrico y oxidación con ácido nítrico; el montaje se realizó en glicerinagelatina. Las láminas (slides o preparados palinológicos) están depositadas en la colección CTES-PMP (Corrientes Preparados Micropaleontológicos) de la Facultad de Ciencias Exactas y Naturales y Agrimensura de la Universidad Nacional del Nordeste (UNNE). Las mismas (preparados palinológicos) fueron analizadas con un microscopio óptico Leitz Diaplan y para las fotografías se utilizó una Cámara digital Canon Power Shot 550; las del microscopio electrónico de barrido, se tomaron con el equipo Jeol de la Universidad Nacional del Nordeste.

Con el fin de lograr la afinidad botánica se comparó el material fósil con actual a partir de láminas de referencia que pertenecen a la Palinoteca Corrientes (PAL-CTES), colección de la Universidad Nacional del Nordeste; la mayoría los botones florales fueron obtenidos del Herbario CTES.

Considerando las novedades obtenidas en afinidades botánicas (géneros y/o familias) se prestó particular atención al registro de ellos en el Cenozoico, desde el Paleoceno hasta el Plioceno inclusive.

\section{SISTEMÁTICA PALINOLÓGICA}

\author{
Clase Tricolpatae Iversen \& Troels-Smith, 1950 \\ Género Spinitricolpites Scholtz, 1985
}

Especie tipo. Spinitricolpites jennerclarkei Scholtz, 1985.

Spinitricolpites anzoteguii sp. nov. (Figuras 2I-L)

Etimología. Dedicado a la paleobotánica Luisa M. Anzótegui. Diagnosis. Grano de polen de ámbito circular. Tricolpado. Exina tectada equinulada.

Diagnosis. Pollen grain with circular amb. Tricolpate. Exine tectate echinulate.

Holotipo. PMP-CTES 2268e: 38,2/98. (Número de lámina de la colección y número de coordenadas del microscopio óptico Leitz Diaplan). Figura 2I. 
Medidas del Holotipo. Diámetro ecuatorial $56 \mu \mathrm{m}$; exina $2 \mu \mathrm{m}$ de espesor.

Paratipo. PMP-CTES: 2268f: 32,5/97,1. (Número de lámina de la colección y número de coordenadas del microscopio óptico Leitz Diaplan). Figura 2J.

Localidad tipo. km 107, Formación San José, noroeste de la Provincia de Tucumán.

Edad. Mioceno medio.

Material adicional. CTES-PMP km 107 2783a: 37,1/97,8; 2268c 47,8/106,8;2783b: 45,8/97,8;40/107,5;2268e: 38,2/98;35,9/103,5; 33,2/104; 2268f: 32,5/97,1;44,5/104,1;38/107,5;2268g: 34,2/107. Quebrada de Jujuil 2569: 45,3/109; 38,4/101,2; 49,5/92,3; 48,2/ 101; 68,3/79,3; 38,2/105,7. (Número de lámina de la colección y número de coordenadas del microscopio óptico Leitz Diaplan).
Descripción. Granos de polen de 52 (61) $70 \mu \mathrm{m}$ de diámetro polar y 44 (55) $66 \mu \mathrm{m}$ de diámetro ecuatorial; prolatos esferoidales, ámbito circular, isopolares, radiosimétricos. Tricolpados, colpos estrechos de 26-38 $\mu \mathrm{m}$ de largo, extendiéndose casi hasta los polos. Exina tectada de $2 \mu \mathrm{m}$ de espesor total, sexina de $1,5 \mu \mathrm{m}$ y nexina de $0,5 \mu \mathrm{m}$. Columelas y téctum conspicuos. Exina espinulada, con el MEB se distingue que el téctum tiene espínulas y perforaciones anuladas, distribuídas irregularmente. Espínulas de 1,1-1,2 $\mu \mathrm{m}$ de alto, separadas por 1,5-2,5 $\mu \mathrm{m}$. Perforaciones de $0,1-0,2$ $\mu \mathrm{m}$ de diámetro con engrosamientos anulares de $1,5 \mu \mathrm{m}$ de diámetro aproximadamente.

Comparaciones. La forma original de estos granos está distorsionada posiblemente debido a la longitud de los

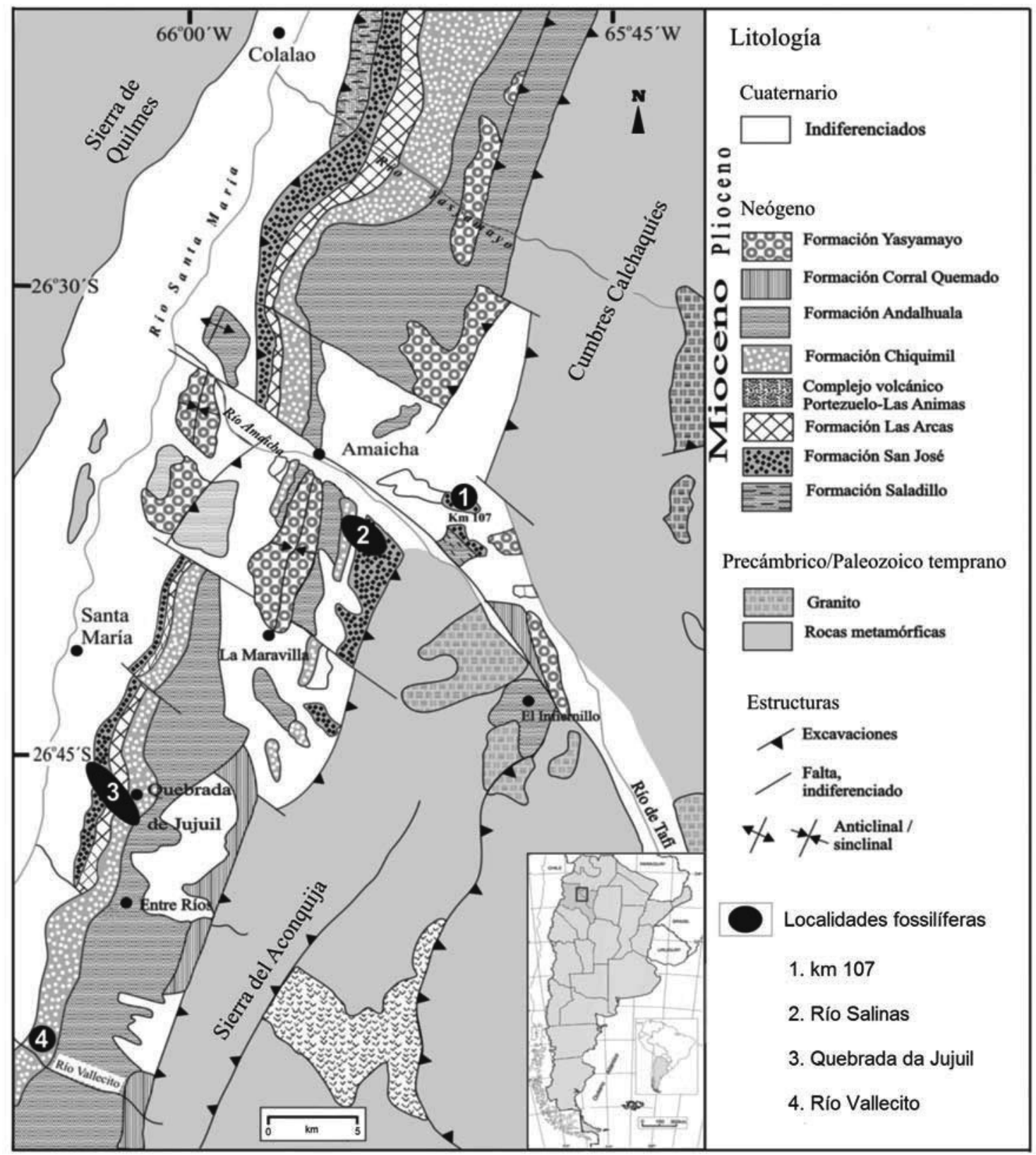

Figura 1. Mapa de ubicación del área de estudio.

Figure 1. Location map of the study area. 
colpos, que producen roturas a manera de gajos. Entre los ejemplares fósiles se ha observado uno que posee más de tres colpos, posiblemente seis. Leuenberger (1976), consideró que este carácter puede ser una variabilidad de Trichocereus (A. Berger) Riccob. (género actual al que es afin el fósil) ya que encontró granos hexacolpados.

La especie tipo Spinitricolpites jennerclarkei se diferencia de esta forma porque es de menor diámetro ecuatorial (39-46 $\mu \mathrm{m})$, las espinas son de mayor dimensión (3-5 $\mu \mathrm{m})$, además carece de espínulas y perforaciones. Scholtz (1985) la relaciona botánicamente a ciertas especies de las Valerianaceae o Verbenaceae.

Spinitricolpites latispinosus (McIntyre) Mildenhall \& Pocknall, 1989 posee mayor espesor de la exina (hasta 2,5 $\mu \mathrm{m}$ ) y de las espinas (2,7 $\mu \mathrm{m}$ de alto y 1,8 a $3,3 \mu \mathrm{m}$ de base) aunque comparte con la especie nueva, el tamaño de los granos.

Spinitricolpites anzoteguii sp. nov. es semejante a cf. Echinocereus sp. y cf. Cereus (Cactaceae), ilustradas en Barnett (1989) para el Mioceno temprano a medio de California. Ambas especies son tricolpadas y con estructura y ornamentación similares; particularmente a cf. Cereus porque tiene elementos positivos sobre el téctum. Dichas especies carecen de descripción por lo que no se pueden hacer comparaciones más precisas.

También es semejante a Echitricolpites giganteus Van der Hammen (1956) del Cuaternario de Colombia por la estructura de la exina (tectada-perforada) y por la ornamentación (equinada); aunque el tamaño general de los granos $(105 \mu \mathrm{m})$, la altura de las espinas $(2,5 \mu \mathrm{m})$ y el espesor de la exina $(3,8 \mu \mathrm{m})$ son mayores. La afinidad botánica que establece este autor para la especie citada es Phyllocactus phyllanthus (L.) Link (Cactaceae). Van der Hammen (1956) crea el morfogénero Echitricolpites, que constituye un subgénero de Tricolpites Cookson ex Couper emend. Jarzen $\&$ Dettmann (1989), basado en una especie actual, por lo cual se considera "nomen nudum" y no se lo utiliza para designar a la nueva especie.

Al comparar esta especie con material palinológico actual ha resultado que varias especies de Cactaceae de la subfamilia Cactoideae tienen las mismas características morfológicas, entre ellas: Trichocereus cabrerae Kiesling, T. strigosus (Salm-Dyck) Britton \& Rose, T. candicans (Gillies ex SalmDyck) Britton \& Rose, T. tarijensis (Vaupel) Werderm. Echinopsis leucantha (Gillies ex Salm-Dyck) Walp, E. aurea Britton \& Rose, E. tubiflora (Pfeiff.) Zucc. y E. rhodotricha K. Schum.

Afinidad botánica. Cactaceae, (Cactoideae), Trichocereus spp., Echinopsis spp.

Distribución y ambiente de los taxones actuales afines. El área de distribución de ambos géneros se superpone aunque sólo marginalmente, Echinopsis Zucc., se extiende desde el océano Atlántico hasta el pie de los Andes mientras que Trichocereus (A. Berger) Riccob., es casi exclusivamente andino y llega hasta las Sierras Pampeanas (centro-oeste) (Kiesling, 1978).

En el género Echinopsis la mayoría de las especies son hierbas y subarbustos suculentos, mientras que las de
Trichocereus son arbustos con tallos cilíndricos y suculentos, generalmente forman matas (Trichocereus cabrerae, $T$. strigosus, T. candicans y T. tarijensis) de hasta $2 \mathrm{~m}$ de altura y 3-4 m de diámetro, en terrenos más o menos horizontales o lomadas en cerros bajos de la precordillera y en las laderas de montañas muy secas. Integran la provincia fitogeográfica del Monte y Prepuneña (Cabrera, 1976 y Kiesling, 1978).

\section{Clase Tetracolpatae Iversen \& Troels-Smith, 1950 Género Retitetracolpites Mathur, 1966}

Especie tipo: Retitetracolpites brevicolpatus Mathur, 1966. Retitetracolpites columelae sp. nov. (Figuras 2G, H, M; 3A)

Etimología. El nombre alude a las columelas libres distribuídas en los lúmenes.

Diagnosis. Grano de polen de ámbito subcircular. Tetracolpado. Exina semitectada con retículo heterobrochado, muros con espínulas, lúmenes con columelas libres (multicolumelados).

Diagnosis. Pollen grain, isopolar, radiosimetric, subcircular amb. Tetracolpate. Exine semitectate with heterobrochate reticulum, walls with spinula, lumina with free columellae (multicolumellate).

Holotipo. CTES-PMP 2268a: 39,9/93,8. (Número de lámina de la colección y número de coordenadas del microscopio óptico Leitz Diaplan). Figura 2G.

Medidas del Holotipo. Diámetro $42 \mu \mathrm{m}$; exina $3 \mu \mathrm{m}$ de espesor. Paratipo. CTES-PMP 1952b: 38,5/107,6. (Número de lámina de la colección y número de coordenadas del microscopio óptico Leitz Diaplan). Figura 2H.

Localidad tipo. km 107, Quebrada de Amaicha, Formación San José, Provincia de Tucumán.

Edad. Mioceno medio.

Material adicional. CTES-PMP km 1072783a: 39,9/93,8; 2268c: 42,6/92,8; 1952b: 38,5/107,6; 2265d: 42/97,2; 2268c: 39,5/104,5. Río Salinas 2839a: 38,4/92,3;42,1/92,3; 2238f: 50/99,1. Quebrada de Jujuil 2581:33,2/101,3; 40,7/83,4; 68,3/107,2; 83,4/92,3; 49,3/ 101,2. (Número de lámina de la colección y número de coordenadas del microscopio óptico Leitz Diaplan).

Descripción. Granos de polen de $30(37,5) 45$ um de diámetro, isopolares, radiosimétricos, posiblemente oblatos, ámbito subcircular. Tetracolpados, colpos de 10-15 $\mu \mathrm{m}$ de largo y 2$4 \mu \mathrm{m}$ de abertura máxima. Exina semitectada, de 2,5-3 $\mu \mathrm{m}$ de espesor total, sexina de 1,5-2 um y nexina de 0,7-1 $\mu \mathrm{m}$. Retículo de muros simplibaculados, fragmentados de 1-1,5 $\mu \mathrm{m}$ de espesor, con elementos positivos, posiblemente espínulas, heterobrochados lúmenes de 2-4 $\mu \mathrm{m}$ de diámetro, poseen columelas libres distribuídas en toda la superficie (multicolumelados), columelas de 2-3 $\mu \mathrm{m}$ de alto. MEB: Confirma la existencia de columelas libres en los lúmenes y que los muros son fragmentados y microequinulados.

Comparaciones. La especie tipo Retitetracolpites brevicolpatus tiene menor tamaño $(28 \mu \mathrm{m})$ y en el retículo no se describen mayores detalles. Retitetracolpites sp. Jarzen 

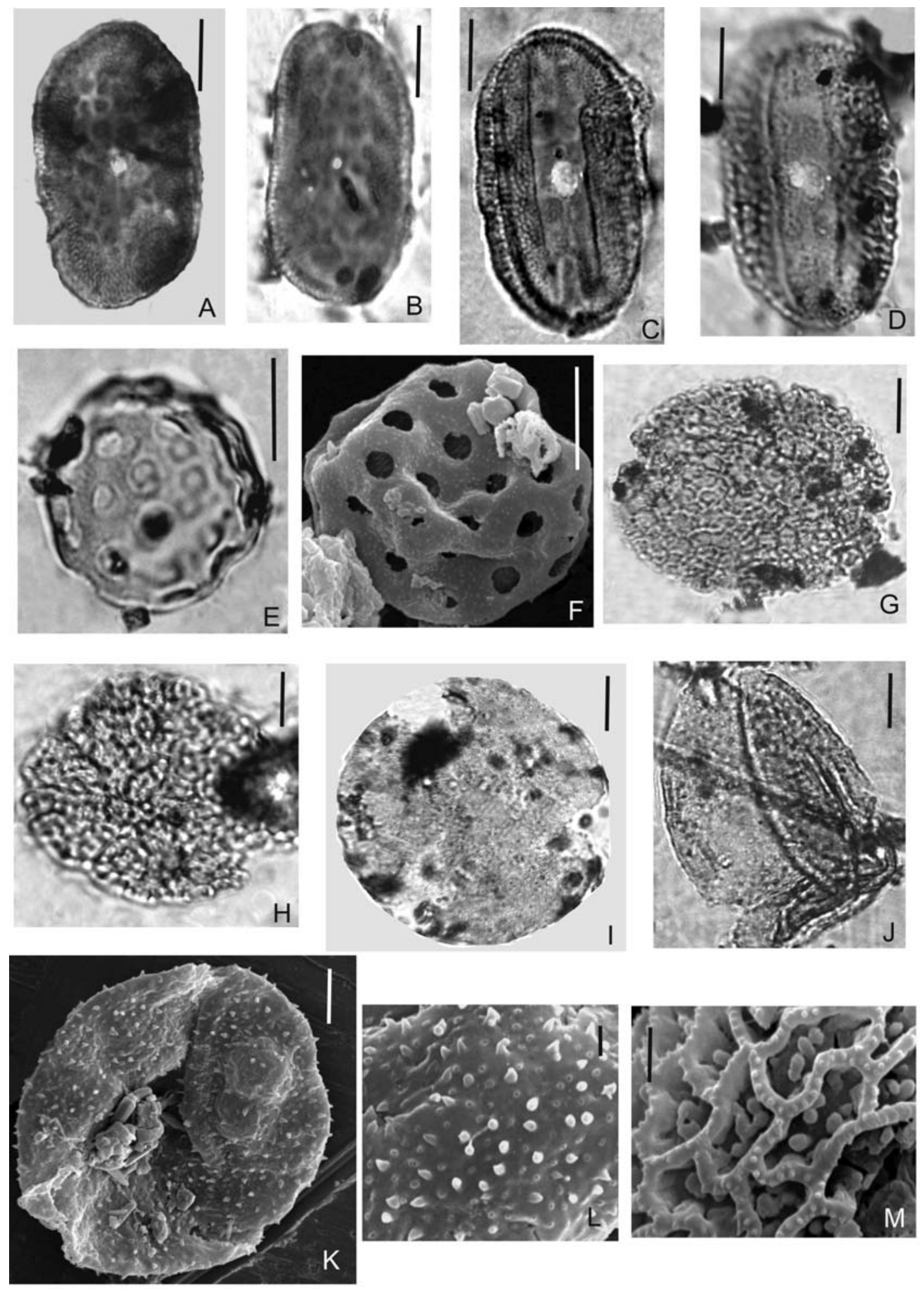

Figura 2. A, B, Areolipollis pirenii sp. nov.: A, vista general, CTES-PMP 2265c 38,1/110,5, holotipo; B, CTES-PMP 2268d - 47/105, 4, paratipo. C, D, Areolipollis insularis sp. nov.: C, CTES-PMP 2598b - 39,4/107,3, holotipo; D, CTES-PMP 1946b - 48,4/102, paratipo. E, F Chenopodipollis minima sp. nov.: E, CTES-PMP 2783 a - 36,5/97,8, holotipo; F, MEB CTES-PMP 2268. G, H, M, Retitetracolpites columelae sp. nov.; G, CTES-PMP 2268 a - 39,9/93,8, holotipo; H, CTES-PMP 1952 b - 38,5/107,6, paratipo; M, detalle de exina MEB CTES-PMP 2268. I-L, Spinitricolpites anzoteguii sp. nov.: I, vista polar CTES-PMP 2268 e - 38,2/98, holotipo; J, vista subecuatorial CTES-PMP 2268 f - 32,5/97,1, paratipo; K, vista polar MEB CTESPMP 2268; $\mathbf{L}$, detalle de ornamentación MEB CTES-PMP 2268. Escalas $=10 \mu \mathrm{m}$, excepto en $\mathbf{L}$ y $\mathbf{M}=2 \mu \mathrm{m}$.

Figure 2. A, B, Areolipollis pirenii sp. nov.: A, general view, holotype; B, paratype. C, D, Areolipollis insularis sp. nov.: C, holotype; D, paratype. E, F, Chenopodipollis minima sp. nov.: E, holotype; F, SEM. G, H, M, Retitetracolpites columelae sp. nov.: G, holotype; H, paratype; M, exine detail SEM. I-L, Spinitricolpites anzoteguii sp. nov.: I, polar view, holotype; J, sub-equatorial view, paratype; K, polar view same paratype; $\mathbf{L}$, detail of ornamentation same paratype. Scale bars $=10 \mu \mathrm{m}$, except in $\mathbf{L}$ and $\mathbf{M}=2 \mu \mathrm{m}$. 
\& Dilcher 2006 del Eoceno de Norteamérica presenta similar tamaño, tres a cuatro colpos y exina finamente reticulada sin embargo carece de los lúmenes multicolumelados típicos de esta nueva forma.

Retitetracolpites columelae sp. nov. es muy parecida a la especie actual Bougainvillea campanulata Heimerl., Nyctaginaceae (Caccavari, 1979) por la forma, tamaño, número de aberturas y características de la exina. Las fotomicrografias con el microscopio electrónico de barrido permitieron corroborar los detalles de los muros, lo que facilitó la relación del fósil con el taxón actual.

Los atributos notorios de estos especímenes permiten ubicarlos en una especie nueva y relacionarlos botánicamente.

Afinidad botánica. Nyctaginaceae, Bougainvillea campanulata Heimerl.

Distribución y ambiente de los taxones actuales afines. La familia Nyctaginaceae está compuesta por 30 géneros y 300 especies que se distribuyen principalmente en regiones tropicales y subtropicales del nuevo y viejo mundo. Pocas especies se encuentran en climas templados (Cronquist, 1981). En la Argentina cuenta con nueve géneros y 14 especies (Zuloaga \& Morrone, 1999). Bougainvillea campanulata ("coronillo") es un arbusto o árbol espinoso, xerófilo, de hasta $6 \mathrm{~m}$ de altura del norte de Argentina (Chaco, Formosa, Jujuy, Tucumán, Salta y Santiago del Estero). Integra el Dominio Chaqueño y relaciona las provincias Chaqueña y del Monte con las del resto del Dominio, junto a Prosopis L. y Bulnesia Gay entre otros géneros (Cabrera, 1976; Lucena, 1993; Toursarkissian, 1975; Zuloaga \& Morrone, 1999).

Clase Diporatae Iversen \& Troels-Smith, 1950 Género Areolipollis Clarke \& Frederiksen, 1968

Especie tipo. Areolipollis ordinus Clarke \& Frederiksen, 1968.

Observaciones. Los géneros Areolipollis Clarke \& Frederiksen (1968) y Multiareolites Germeraad, Hopping \& Muller (1968), fueron creados en el mismo año, coincidentemente para granos fósiles de morfología similar: simetría bilateral, dicolporados-dicolpados con areolas o engrosamientos sexínicos circulares (aquí descriptas como islas de la zona apertural) en hileras o dispuestas irregularmente, mesocolpios reticulados. Estos morfogéneros fueron relacionados con taxones de la actual familia Acanthaceae (Justicia, Jacobina, Beloperone, Dianthera, Anisotus, Rungia). Multiareolites surge con el fin de reemplazar a Dicolporites formosus Van der Hammen (1956) que tenía como especie tipo a una especie actual (Beloperone bracteosa, Acanthaceae). Germeraad et al. (1968), realizan una nueva combinación y adoptan a Dicolporites formosus Van der Hammen como base para dicha combinación. Jansonius \& Hill (1976) señalan que esta nueva combinación es incorrecta, ya que está basada en polen actual.

Dada la confusión originada entorno al morfogénero Multiareolites, en este trabajo, se considera conveniente de que los granos de polen fósiles, dicolporados-diporados, con una o más hileras de islas a ambos lados de la apertura y con afinidad al género Justicia (Acanthaceae), se refieran al morfogénero Areolipollis Clarke \& Frederiksen, 1968. Es necesario aclarar que tanto en las diagnosis de Areolipollis como en la de Multiareolites no está contemplada la apertura diporada; no obstante con el estudio de las especies actuales de Justicia (Pire et al., 2006), quedó demostrado, que pueden tener aberturas di-colporadas o di-poradas (con frecuencia relacionadas a colpoides o a surcos, vistos con el MEB).

Los registros de Acanthaceae fósiles afines al género Justicia se encuentran en: el Neógeno de México (Elsik, 1969); Venezuela, Oligoceno-Mioceno (Lorente, 1986) у Plioceno-Cuaternario (Müller et al., 1987); Caribe y Nigeria (Multiareolites formosus) en el Mioceno-Plioceno (Germeraad et al., 1968); en el Mioceno de Brasil (Regali et al., 1974), y en este mismo país, en el Oligo-Mioceno (Yamamoto, 1995 como Areolipollis sp.1 y Areolipollis sp. 2).

\section{Areolipollis pirenii sp. nov. (Figuras 2A, B)}

Etimología. El nombre de la especie hace alusión a la palinóloga Stella M. Pire.

Diagnosis. Grano de polen prolato, isopolar, con simetría bilateral. Diporado. Dos áreas aperturales rodean a las aberturas y dos áreas no apertuales se sitúan entre ambas. En la sección media de cada área apertural se encuentra un surco marcado. El área apertural contiene cuatro hileras de islas sexínicas, pequeñas y de disposición desordenada. Exina en el área apertural psilada a rugulada y en el área interapertural reticulada, ligeramente más engrosada en el área ecuatorial. Diagnosis. Pollen grain prolate, isopolar, with bilateral symmetry. Diporate. Two apertural area surround to the aperture and two non apertural areas are placed between both. In the midway section of each apertural area is a pronounced furrow. The apertural area contains four rows of sexine small islands of disorderly disposition. Exine psilate to rugulate in the apertural area and in the reticulate interapertural area, slightly more thickened in the equatorial area.

Holotipo. CTES-PMP 2265c 38,1/110,5. (Número de lámina de la colección y número de coordenadas del microscopio óptico Leitz Diaplan). Figura 2A.

Medidas del Holotipo. Diámetro polar $38 \mu \mathrm{m}$; diámetro ecuatorial $24 \mu \mathrm{m}$; exina $1,4 \mu \mathrm{m}$ en los polos y $2,1 \mu \mathrm{m}$ en el ecuador.

Paratipo. CTES-PMP 2268d: 47/105, 4. (Número de lámina de la colección y número de coordenadas del microscopio óptico Leitz Diaplan). Figura 2B.

Localidad tipo. km 107, Formación San José, noroeste de la Provincia de Tucumán.

Edad. Mioceno medio.

Material adicional. CTES-PMP km 107 2265c: 46/99,8; 58,9/ 103,1;56,3/99; 44,6/98,4; 57,8/100,3; 1946 b: 38,5/104,1; 1946d: 44/107,2; 2269a: 47,3/96,4. Río Salinas 2223a: 38,5/101,5; 39,4/ 105,4. 2839 a: 40/92,3; 39,2/92,3. (Número de lámina de la colección y número de coordenadas del microscopio óptico Leitz Diaplan).

Descripción. Granos de polen de 30 (36) 42 um de diámetro 
polar por $21(22,5) 24 \mu \mathrm{m}$ de diámetro ecuatorial, prolatos, isopolares, con simetría bilateral. Diporados. Las zonas que rodean a las aperturas se denominan áreas aperturales y áreas no aperturales a las que se sitúan entre ambas. En la sección media de cada área apertural se encuentra el poro de forma más o menos circular de 2-3,5 $\mu \mathrm{m}$ de diámetro y un surco central marcado (25-28 $\mu$ m aproximadamente de largo). El área apertural contiene 4 hileras de islas reticuladas, dos a cada lado de la abertura, de disposición desordenada, cada hilera lleva aproximadamente ocho a diez islas reticuladas, de 1,4$2,1 \mu \mathrm{m}$ de diámetro cada una.

La exina tiene 1,4 $\mu \mathrm{m}$ de espesor en los polos (sexina y nexina de igual espesor, $0,7 \mu \mathrm{m}$ cada una) y está ligeramente más engrosada en el área ecuatorial no apertural, donde llega a medir $2,1 \mu \mathrm{m}$ (sexina 0,7 a $1,4 \mu \mathrm{m}$ y nexina de $0,7 \mu \mathrm{m}$ ). La sexina del área apertural es psilada a rugulada en cambio en el área no apertural es reticulada, con lúmenes cercanos a los $0,5 \mu \mathrm{m}$ de diámetro.

Comparaciones. Se ha observado un ejemplar con colpoides. Areolipollis pirenii sp. nov. se diferencia de la especie tipo Areolipollis ordinus Clarke \& Frederiksen porque es diporada y posee cuatro hileras de islas, en cambio la especie tipo es dicolporada, con dos hileras de islas. Areolipollis sp. 1 y A. sp. 2, en Yamamoto (1995) también son dicolporadas y la exina en el ecuador alcanza los $7 \mu \mathrm{m}$ de espesor.

Areolipollis pirenii sp. nov. es similar al tipo polínico actual Justicia corumbensis, subtipo polínico J. dumetorum (Pire et al., 2006) por la presencia de aberturas diporadas, surco central, número y disposición de las islas; no obstante por las cuatro hileras de islas de disposición desordenada la especie fósil es más parecida a J. brasiliana Roth, que se encuentra en el mismo tipo polínico.

Afinidad botánica. Acanthaceae, Justicia brasiliana Roth. Distribución y ambiente de los taxones actuales afines. El género Justicia es el más grande y complejo de la familia, muy diversificado en regiones tropicales y subtropicales de América del Sur. En la Argentina reúne 27 especies que son hierbas y arbustos perennifolios (Graham, 1988).

Justicia brasiliana es un arbusto que se encuentra en bosques húmedos o con suelos bien drenados del noreste de Argentina: Corrientes (en bosques higrófilos del Iberá), Misiones (Parque Provincial Teyú Cuaré) y en Paraguay oriental (Ezcurra, 2002; Biganzoli \& Múlgura de Romero, 2004; Tressens et al. in Arbo \& Tressens, 2002).

Areolipollis insularis sp. nov. (Figuras 2C, D)

1997 Areolipollis sp. Mautino, Anzótegui \& Herbst, p. 127, fig. 4.

Etimología. El nombre está referido a las islas sexínicas de las áreas aperturales.

Diagnosis. Grano de polen, prolatos, isopolar, con simetría bilateral. Diporado. Dos áreas aperturales rodean a las aberturas y dos áreas no aperturales se sitúan entre ambas. En la sección media de cada área apertural se encuentra un surco marcado. El área apertural contiene dos hileras de islas sexínicas. Exina más engrosada en el área ecuatorial que en los polos.

Diagnosis. Pollen grain prolate, isopolar, with bilateral symmetry. Diporate. Two apertural areas surround to the aperture and two non apertural areas are placed between both. In the midway section of each aperturate area is a marked furrow. The apertural area contains two rows of sexine islands. Exine more thickened in the equatorial area that in the poles. Holotipo. CTES-PMP 2598b: 39,4/107,3. (Número de lámina de la colección y número de coordenadas del microscopio óptico Leitz Diaplan). Figura 2C.

Dimensiones del Holotipo. Diámetro polar 40,2 $\mu \mathrm{m}$; diámetro ecuatorial $22 \mu \mathrm{m}$; exina $2 \mu \mathrm{m}$ de espesor los polos y $4 \mu \mathrm{m}$ en el ecuador.

Paratipo. CTES-PMP 1946b: 48,4/102. (Número de lámina de la colección y número de coordenadas del microscopio óptico Leitz Diaplan). Figura 2D.

Localidad tipo. km 107, Formación San José, noroeste de la Provincia de Tucumán.

Edad. Mioceno medio.

Material adicional. CTES-PMP km 107 1946b: 48,4/102; 38,9/ 104,$9 ; 98 / 101,5 ; 45,8 / 99,2 ; 2268 \mathrm{c}: 37,4 / 107$. Quebrada de Jujuil 2578: 39,4/102,4;56,3/99,2;35,4/105;48,3/96,5;2541:39,4/103. (Número de lámina de la colección y número de coordenadas del microscopio óptico Leitz Diaplan).

Descripción. Granos de polen de 39 (40,5) $42 \mu \mathrm{m}$ de diámetro polar por $21(22,5) 24 \mu \mathrm{m}$ de diámetro ecuatorial, bilaterales, prolatos, isopolares. Diporados. Las zonas que rodean a las aperturas se denominan áreas aperturales y áreas no aperturales a las que se situan entre ambas. En la sección media de cada área apertural, se ubica el poro y perpendicuarmente a éste, un surco central que llega hasta los polos. Poros subcirculares de 2,8-5 $\mu \mathrm{m}$. El área apertural contiene dos hileras de islas reticuladas y bajas (una a cada lado de la abertura), cada hilera con cinco a seis islas de 2-2,8 $\mu \mathrm{m}$ de diámetro, ubicadas ordenadamente. Exina tectada, microrreticulada; notablemente de mayor espesor en el ecuador $(4-4,5 \mu \mathrm{m}$ de espesor total, sexina con capa columelar de 1-1,5 um y téctum de 1,5 $\mu \mathrm{m}$; nexina de 1-1,5 $\mu \mathrm{m}$ ) que en los polos ( $2 \mu \mathrm{m}$ espesor total, sexina y nexina de $1 \mu \mathrm{m}$, cada una). Comparaciones. Areolipollis pirenii sp. nov., se diferencia de Areolipollis insularis sp. nov. porque tiene cuatro hileras de islas dispuestas desordenadamente; ambas especies comparten el tamaño más o menos similar del grano, el tipo de aperturas y la exina más desarrollada en la zona ecuatorial. También se diferencian de Multiareolites formosus (Van der Hammen, 1956) Germeraad et al. 1968 y Areolipollis sp. 1 (in Yamamoto, 1995) porque son dicolporados; aunque se parecen porque tienen similares tamaños de los granos, dos hileras de islas y la exina marcadamente más engrosada en el ecuador.

En Graham (1976) del Mioceno de México se ilustran dos tipos de Justicia sp., que aunque no se describen, comparten los mismos caracteres con Areolipollis insularis sp. nov.

La especie fósil aquí descripta es similar al tipo polínico actual Justicia corumbensis, subtipo polínico J. dumetorum (Pire et al., 2006) en especial al subtipo polinico J. laevilinguis 
que comprende a esta única especie por el tipo de aperturas, tamaño del grano y características de la exina. Especialmente por la presencia de dos hileras de islas sexínicas a cada lado de las aperturas en el área apertural.

Afinidad botánica. Acanthaceae, Justicia laevilinguis (Nees) Lindau.

Distribución y ambiente de los taxones actuales afines. Justicia laevilinguis es una hierba que crece en campos, ambientes anegados o inundables presentes en Tucumán, Formosa, Entre Ríos, Chaco y Buenos Aires (Ezcurra, 2002). Se la halló también en los esteros del Iberá (Arbo \& Tressens, 2002).

\section{CLASE PERIPORATAE Iversen \& Troels Smith, 1950 Género Chenopodipollis Krutzsch, 1966}

Especie tipo. Chenopodipollis multiplex (Weyland \& Pflug) Krutzsch, 1966.

Chenopodipollis minima sp. nov.

(Figuras 2 E, F)

1997 Polyporina sp. Mautino, Anzótegui \& Herbst, p. 127, fig. 4.

2002b Psilaperiporites cf. P. minimus Regali, Uesugui \& Santos. Mautino \& Anzótegui, p. 274, fig. 1G.

1986 Chenopodiipollis sp. 2. Anzótegui \& Garralla, p. 13, lám. 6, figs. 81, 82.

Etimología. El nombre de la especie está referido al tamaño de los granos.

Diagnosis. Grano de polen apolar, radiosimétrico, esferoidal. Periporado. Exina tectada, escabrada.

Diagnosis. Pollen grains apolar, radiosimetric, spheroidal. Periporate. Exine tectate, scabrate.

Holotipo. CTES-PMP 2783a: 36,5/97,8. (Número de lámina de la colección y número de coordenadas del microscopio óptico Leitz Diaplan). Figura 2E.

Dimensiones del Holotipo. Diámetro $21 \mu \mathrm{m}$; número de poros 34; diámetro 2,5 $\mu \mathrm{m}$; exina de $1 \mu \mathrm{m}$ de espesor.

Paratipo. CTES-PMP 2265c: 40,1/98,7. (Número de lámina de la colección y número de coordenadas del microscopio óptico Leitz Diaplan).

Localidad tipo. km 107, Formación San José, noroeste de la Provincia de Tucumán.

Edad. Mioceno medio.

Material adicional.CTES-PMP Río Vallecito 1694f: 38,5/107,8; 1695d: 34,3/98,2;34,2/99; 1695k: 39,1/95,8; 1795a:46/108; 1796a: 39,2/107,9; 1798a: 37,9/109,5; 1801b: 40,5/94,4; 1801d: 36/109,6; 37,5/111,6; 1920b: 42/98,5; 1920c: 43,5/100,2; 1795c: 34,1/99,7; 1695g: 36,8/95. Y otros en la Localidad km 107 (diez ejemplares), Río Salinas (13 ejemplares) y Quebrada de Jujuil (18 ejemplares). (Número de lámina de la colección y número de coordenadas del microscopio óptico Leitz Diaplan).

Descripción. Granos de polen de 16 (19) $22 \mu \mathrm{m}$ de diámetro, radiosimétricos, esferoidales, apolares, contorno sinuoso, periporados. Poros de forma circular, en número 30-40, poros con diámetro de $2-3 \mu \mathrm{m}$, y pequeños anillos de $0,5 \mu \mathrm{m}$. Exina tectada de 1-1,5 $\mu \mathrm{m}$ de espesor total, sexina de $1 \mu \mathrm{m}$ a menos y nexina de $0,5 \mu \mathrm{m}$ o menos, estratificación difícil de distinguir. Téctum escabrado. El MEB revela la presencia de espínulas supratectales.

Comparación. Chenopodipollis chenopodiaceoides (Martin) Truswell et al., 1985 es semejante, pero presenta confusión en la diagnosis en cuanto al número de aberturas. Martin (1973) sinonimiza Polyporina chenopodiaceoides a $C$. chenopodiaceoides y considera a la especie con 25 a 60 poros. Truswell et al. (1985), en la combinación que propone, describe granos con 50-60 poros; desde el momento en que $C$. chenopodiaceoides quedó instaurada, la especie fue registrada en numerosas ocasiones con un número variable de poros, pero dentro del rango establecido por Martin (1973); Barreda (1997) con 30 a 56 poros; en Ottone et al. (1998) con 36-46 poros. No obstante, los registros más abundantes señalan palinomorfos con 50 o 60 poros (cercano al límite superior).

En síntesis, el registro Chenopodipollis chenopodiaceoides concentra un rango de aberturas muy amplio y variable; especialmente si se considera el criterio usado para taxones actuales, afines al Complejo Chenopodiaceae/Amaranthaceae, por el cual varias especies podrían diferenciarse a partir del número de poros (granos periporados con diferencias de 15 poros) sumado al resto de las características.

Por lo tanto en este trabajo, se considera que en Chenopodipollis chenopodiaceoides deben incluirse granos con 45-60 poros y en la especie aquí creada, con 30 a 40 poros.

Chenopodipollis minima es similar al tipo polínico actual Amaranthus, (Amaranthaceae) Cuadrado, en Pire et al. (1998), pero intermedia a los subtipos Amaranthus muricatus y Chamissoa acuminata. Con el primero comparte el mismo tamaño de los granos, número de poros y espesor de la exina, con el segundo el diámetro de los poros. Sin embargo, también puede vincularse a la especie nueva, aquí descripta, con la familia Chenopodiaceae dado que algunos taxones resultan muy parecidos como Atriplex lampa Gill. ex Moq. y Suaeda divaricata Moq. (Markgraf \& D'Antoni, 1978).

Afinidad botánica. Complejo Amaranthaceae/Chenopodiaceae. Distribución y ambiente de los taxones actuales afines. Las especies que están incorporadas en los subtipos polínicos Amaranthus muricatus y Chamissoa acuminata de Amaranthaceae, son hierbas y arbustos de sabanas y abundantes en el centro y norte de la República Argentina (Covas, 1941; Hauman, 1924 y Sohmer, 1976). Las Chenopodiaceae también son hierbas o arbustos de amplia distribución mundial aunque confinadas en áreas xéricas o salitrosas en estepas con suelos salinos o en terrenos bajos con agua salada. Muchas especies muestran adaptaciones xerofíticas (Giusti, 1987; Heywood in Yamamoto, 1995).

Género Periporopollenites Pflug \& Thomson en Thomson \& Pflug, 1953

Especie tipo. Periporopollenites stigmosus Thomson \& Pflug, 1953.

Observaciones. El género Periporopollenites Pflug \& Thomson (Thomson \& Pflug, 1953) incluye formas periporadas con diez a 
32 poros, con o sin membrana apertural, exina punctadogranulada a finamente reticulada con afinidad a Caryophyllaceae. Caryophyllidites Couper 1960 (más de 20 poros no anillados), con posible afinidad a Caryophyllaceae, fue pasado a sinonimia de Periporopollenites en Stover \& Partridge (1973); aunque Anzótegui \& Cuadrado (1996) lo relacionan con el complejo Amaranthaceae-Chenopodiaceae. Periporopollenites difiere de Chenopodiipollis Krutzsch, 1966 no solo por poseer menor número de poros sino también por la presencia de una exina más gruesa.

Con estos antecedentes queda demostrada la amplia afinidad botánica del género Periporopollenites: con la familia Caryophyllaceae, con el complejo Amaranthaceae-Chenopodiaceae (cuando los granos poseen un número de poros menor o igual a 32) y en este trabajo se incorpora su relación con las Cactaceae.

\section{Periporopollenites vivianae sp. nov.}

(Figuras 3B-E)

Etimología. Está dedicado a la palinóloga Viviana Barreda. Diagnosis. Grano de polen apolar, oblato-esferoidal, ámbito subcircular. Periporado. Exina semitectada, reticulada.

Diagnosis. Pollen grain apolar, oblato-spheroidal, subcircular amb. Periporate. Exine semitectate, reticulate.
Holotipo. CTES-PMP 2783a: 46/100,5. (Número de lámina de la colección y número de coordenadas del microscopio óptico Leitz Diaplan). Figuras 3C, D.

Dimensiones del Holotipo. Diámetro $80 \mu \mathrm{m}$; número de poros 14; diámetro de los poros 12-15 $\mu$ m y exina 5 a $6 \mu \mathrm{m}$ de espesor. Paratipo. CTES-PMP 2268c 37/102,6. (Número de lámina de la colección y número de coordenadas del microscopio óptico Leitz Diaplan).

Localidad tipo. km 107, Formación San José, noroeste de la Provincia de Tucumán.

Edad. Mioceno medio.

Material adicional. CTES-PMP km 107 2783a: 46/100,5; 44,3/ 97; 43,7/101,1; 2268c:40/106; 37,4/102,8; 2268e: 34,2/105; 1952b: 40,5/97,3; 1952d: 39,5/200; 2268f: 38,5 1946d: 43/100,6; 1951b: 43,5/101,6. Río Salinas 1705a: 40/96; 36,5/101,2; 39,4/ 103. 2839a: 45,4/105,2; 45/96; 34,7/100; 35,2/99, 1; 39,2/84,5; 2238c: 43,2/104. Quebrada de Jujuil 2570: 46,5/100,8; 45,3/98,5; 34,2/102,3; 39,7/89,8; 43,8/102,939,7/99,4; 48,3/89,2; 39,4/10,9; 29,3/102; 39,4/99,5; 39,4/109,2. (Número de lámina de la colección y número de coordenadas del microscopio óptico Leitz Diaplan).

Descripción. Granos de polen de $65(66,5) 88 \mu \mathrm{m}$ de diámetro, oblato-esferoidales, apolares, ámbito subcircular. Periporados, con 14 a 22 poros de diez a $20 \mu$ m de diámetro, de forma
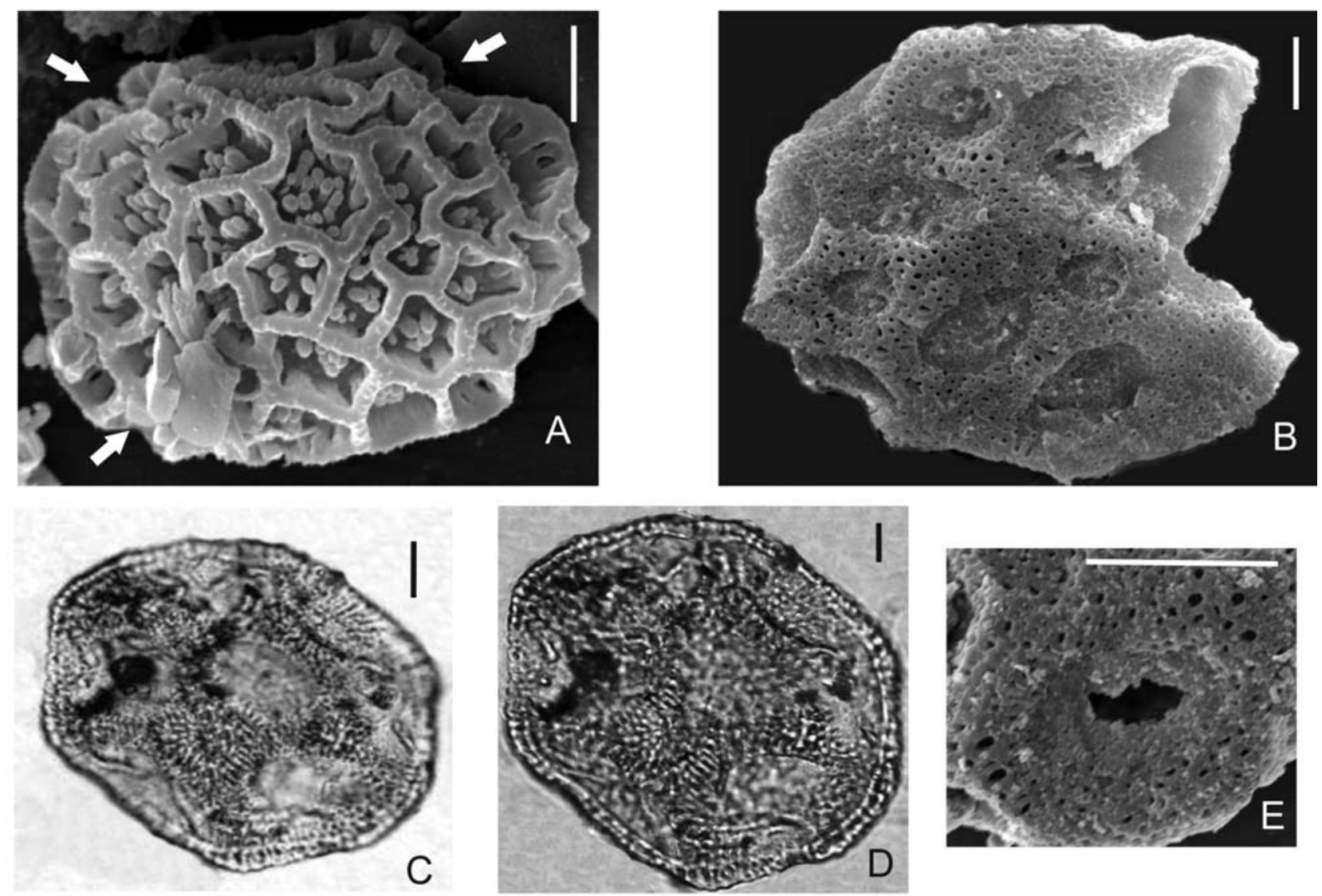

Figura 3. A, Retitetracolpites columelae sp. nov.,vista general MEB, CTES-PMP 2268. B-E, Periporopollenites vivianae sp. nov.: B, MEB CTES-PMP 2268; C, CTES-PMP 2783 a - 46/100,5, holotipo; D, holotipo, otro foco; E, detalle de la apertura al MEB CTES-PMP 2268. Escalas $=10 \mu \mathrm{m}$.

Figure 3. A, Retitetracolpites columelae sp. nov., SEM general view. B-E, Periporopollenites vivianae sp. nov.: B, SEM general view; C, holotype; D, holotype, other focus; E, SEM aperture detail. Scale bars $=10 \mu \mathrm{m}$. 
circular a elíptica, ligeramente hundidos, cubiertos por membrana apertural. Exina semitectada, de 5-6 $\mu \mathrm{m}$ de espesor, adelgazándose hacia las aberturas, sexina 4-5 $\mu \mathrm{m}$ (se adelgaza hacia los poros) y nexina de $1 \mu \mathrm{m}$. Columelas de $1-1,5 \mu \mathrm{m}$ de ancho y cabezuelas, de 1,5-2 $\mu \mathrm{m}$ de diámetro. Exina reticulada, lúmenes de 0,5-0,7 $\mu \mathrm{m}$ de diámetro. El MEB permite corroborar que la exina es reticulada y que los poros conservan membranas aperturales ornamentadas con microgránulos.

Comparaciones. Se ha observado que el contorno de los granos es ondulado debido al diámetro que tienen los poros y al ligero hundimiento que presentan; además estas aberturas exhiben diferentes diámetros en un mismo ejemplar.

Periporopollenites demarcatus Stover, 1973 (en Stover \& Partridge, 1973) difiere por el tamaño (26-35 $\mu \mathrm{m})$, y el número y tamaño de los poros (12 y 6-8 $\mu \mathrm{m}$, respectivamente). Periporopollenites vesicus Patridge, 1973 (en Stover \& Partridge, 1973), posee igual número de poros (14 a 25) aunque menores dimensiones en el diámetro general del grano (23-50 $\mu \mathrm{m})$ y en el espesor de la exina $(1,5-3 \mu \mathrm{m})$ que es punctada-reticulada.

Esta forma fue comparada con varias especies actuales argentinas de Opuntia Mill. (Cactaceae) y comparte mayores similitudes con $O$. aurantiaca Lindl. (PAL-CTES 6978), $O$. chakensis Spegazzini (PAL-CTES 6984), O. ficus-indica L. Miller (PAL-CTES 6987), O. prasina Spegazzini (PAL-CTES 6982) y O. anacantha Spegazzini var. anacantha Kiesling (PAL-CTES 6994).

Afinidad botánica. Cactaceae (Opuntioideae), Opuntia aurantiaca, O. chakensis, O. ficus indica, O. prasina y $O$. anacantha.

Distribución y ambiente de los taxones actuales afines. Los vegetales comprendidos en el género Opuntia son arbustos o subarbustos con tallos comprimidos que viven, generalmente en ambientes áridos y semiáridos del país en bordes o claros de bosques xerófilos de las provincias de Chaco, Entre Ríos, Formosa, Santa Fe, Corrientes, Jujuy, Salta, Santiago del Estero, Córdoba y Buenos Aires. Integran las provincias fitogeográficas: chaqueña, del espinal, prepuneña y del monte (Cabrera, 1976; Kiesling, 1998).

\section{DISCUSIÓN}

De las especies nuevas aquí descriptas se discutirán dos aspectos: taxonómico y paleoambiental. Desde el punto de vista palino-taxonómico constituyen novedad en estratos argentinos paleocénicos-pliocénicos (se acota este lapso, debido a que en el Cuaternario las especies actuales están mayormente representadas y diversificadas) el registro de los siguientes géneros actuales: Trichocereus, Echinopsis y Opuntia, (representados por Spinitricolpites anzoteguii y Periporopollenites vivianae, Cactaceae); Boungainvillea (representado por Retitetracolpites columelae, Nyctaginaceae); y Justicia (representado por Areolipollis pirenii y Areolipollis insularis, Acanthaceae). Cabe destacar que el registro fósil de la familia Cactaceae es absolutamente novedoso en Argentina para el lapso Paleoceno-Plioceno. Con respecto a la familia Nyctaginaceae es pobre en registros fosilíferos; sin embargo fue documentada por granos de polen de Lymingtonia? sp., por Barreda et al. (1998), en el Mioceno de San Juan y Zetter et al. (1999) dan a conocer un género indeterminado en el Eoceno de Tierra del Fuego. El hallazgo de megafósiles, también da cuenta de la presencia de la familia, de tal manera que Berry (1938), cita impresiones de hojas (Nyctaginites ellipticus) en la Formación Auca Pan Río Pichileufú del Paleoceno-Eoceno Río Negro. A la familia Acanthaceae, se la reconoce solamente por el registro polínico de Multimarginites paluster (Anzótegui \& Cuadrado, 1996) afín a Bravaisia Alph. DC., en la Formación Palo Pintado (Mioceno superior). Por el contrario, el complejo ChenopodiaceaeAmaranthaceae, se encuentra ampliamente representado en el lapso establecido y solamente por palinomorfos; entre ellos: Gomphrenipollis Anzótegui \& Cuadrado, Periporopollenites Pflug \& Thomson, Chenopodipollis Krutzsch y Pffafia Mart. (e.g. Anzótegui \& Garralla, 1986; Anzótegui \& Cuadrado, 1996; Barreda, 1997; Barreda et al., 1998; Guerstein et al., 2001; Guler et al., 2001; Palarmaczuk \& Barreda, 2000; Zamaloa, 2000). De ellos, Gomphrenipollis posee afinidad cierta a Gomphrena (son granos semitectados, reticulados con poros en los lúmenes) y Pffafia Mart., en Anzótegui \& Garralla (1986) al género homónimo ambos de la familia de Amaranthaceae. En tanto que Chenopodipollis Krutzsch (1966) está ligado al complejo Chenopodiaceae-Amaranthaceae dado que el polen, por lo general, resulta indistinguible entre estas dos familias (granos pantoporados, poros de número variable, tectados con microgránulos o espínulas). Por último el género Periporopollenites Pflug \& Thomson (in Thomson \& Pflug, 1953) posee afinidad botánica aún más amplia: a Caryophyllaceae (Thomson \& Pflug), al complejo Amaranthaceae-Chenopodiaceae (Anzótegui \& Garralla, 1986), a las Amaranthaceae (Periporopollenites vesicus in Mautino, 2010) y en el presente trabajo se amplía su afinidad a las Cactaceae con la especie Periporopollenites vivianae.

Desde el punto de vista paleoambiental las especies nuevas aquí descriptas habrían integrado tres paleocomunidades vegetales, de acuerdo a los requerimientos de hábito y hábitat de las especies actuales a las cuales son afines los fósiles: (i) hidrófila palustre, en suelos inundados cercanos a cuerpos de agua dulceacuícolas, con hierbas de Areolipollis insularis sp. nov.; (ii) bosques higrófilos con Areolipollis pirenii en el estrato arbustivo; (iii) comunidad xerófila ubicada en lugares más alejados del cuerpo de agua, con Chenopodipollis minima en el estrato herbáceo y Periporopollenites vivianae, Spinitricolpites anzoteguii y Retitetracolpites columelae en el estrato arbustivo-arbóreo.

\section{CONCLUSIÓN}

En esta oportunidad, de la microflora de las formaciones San José y Chiquimil se brindan seis especies nuevas: Spinitricolpites anzoteguii sp. nov., Periporopollenites vivianae sp. nov. (ambas Cactaceae), Retitetracolpites columelae sp. nov. (Nyctaginaceae), Chenopodipollis minima sp. nov. (Complejo Chenopodiaceae-Amaranthaceae), Areolipollis pirenii sp. nov. y A. insularis sp. nov. (ambas Acanthaceae). 
Los registros de los géneros actuales Trichocereus (A. Berger) Riccob., Echinopsis Zucc. y Opuntia Mill. (Cactaceae), Boungainvillea Comm. ex Juss. (Nyctaginaceae) y Justicia L. (Acanthaceae) constituyen las primeras citas para sedimentos del lapso Paleoceno-Plioceno de Argentina. Cabe mencionar que las especies nuevas contribuyen con nuevos componentes a las comunidades vegetales palustres, bosque higrófilo y xerófilo de las formaciones San José y Chiquimil ratificando que se habrían desarrollado bajo un clima cálido con marcada estacionalidad (Kleinert \& Strecker, 2001).

Finalmente, esta contribución enriquece el conocimiento de las paleofloras cenozoicas en especial del Mioceno, época en que se encuentran en radiación un gran número de familias modernas y por ende, los antecesores de los vegetales actuales.

\section{AGRADECIMIENTOS}

La autora agradece a L. Anzótegui por la lectura crítica del manuscrito y las valiosas sugerencias que mejoraron la calidad del trabajo; al R. Herbst por su permanente estímulo al trabajo; al Comité Editor de esta revista y a M. Quattrocchio por sus enriquecedores comentarios, realizados en calidad de árbitros; al L. Pacella por la confección del mapa y a E. Acevedo por la preparación de las muestras.

\section{REFERENCIAS}

Anzótegui, L.M. 2006. Paleofloras del Mioceno en los Valles Calchaquies, Noroeste de Argentina. Universidad Nacional del Nordeste, Tesis Doctoral, $266 \mathrm{p}$.

Anzótegui, L.M. \& Cuadrado, G. 1996. Palinología de la Formación Palo Pintado, Mioceno Superior, provincia de Salta, República Argentina. Parte 1. Taxones nuevos. Revista Española de Micropaleontología, 28:77-92.

Anzótegui, L.M. \& Garralla, S. 1986. Estudio palinológico de la Formación Paraná (Mioceno Superior) (Pozo Josefina, provincia de Santa Fe, Argentina). Parte 1. Descripciones sistemáticas. Facena, 6:101-176

Arbo, M.M. \& Tressens, S.G. 2002. Flora del Iberá. Corrientes, EUDENE/UNNE, $614 \mathrm{p}$.

Barreda, V.D. 1997. Palynomorph assemblage of the Chenque Formation Late Oligocene- Miocene from Golfo San Jorge Basin, Patagonia, Argentina. Part 4. Polycolporate and porate pollen. Ameghiniana, 34(2):145-154.

Barreda, V.D.; Anzótegui, L.M.; Prieto, A.; Aceñolaza, P.; Bianchi, M.; Borromei, A.; Brea, M.; Caccavari, M.; Cuadrado, A.; Garralla, S.; Grill, S.; Guerstein, R.; Lutz, A.; Mancini, V.; Mautino, L.; Ottone, E.; Quattrocchio, M.; Romero, E.; Zamaloa, M. \& Zucol, A. 2007. Diversificación y cambios de las angiospermas durante el Neógeno en Argentina. In: S. Archangelsky; T. Sánchez \& E.P. Tonni (eds.) Ameghiniana $50^{\circ}$, Publicación Especial, 11:173-191.

Barreda, V.D.; Gutiérrez, P.R. \& Limarino, C.O. 1998. Edad y paleoambiente de la "Serie del Yeso", Valle del Cura, Provincia de San Juan: evidencias palinológicas. Ameghiniana, 35(3):321-335.

Barnett, J. 1989. Palynology and Paleoecology of the Tertiary Weaverville Formation, Northwestern California, U.S.A Palynology, 13:195-246.

Berry, E.W. 1938. Tertiary Flora from the Rio Pichileufú, Argentina. Geology Society American, Special Paper, 12:1-149.

Biganzoli, F. \& Múlgura de Romero, M.E. 2004. Inventario florístico del Parque Provincial Teyú Cuaré y alrededores (Misiones, Argentina). Darwiniana, 42(1/4):1-24.

Bossi, G.E.; Gavriloff, I.J.C. \& Esteban, G. 1998. Terciario, Estratigrafía, Bioestratigrafía y Paleogeografía. In: M. Gianfrancisco; N.E. Puchulu; J. Durango de Cabrera \& G.F. Aceñolaza (eds.) Geología de Tucumán. Colegio de Graduados en Ciencias Geológicas de Tucumán, p. 87-108.

Bossi, G.E. \& Palma, R. 1982. Reconsideración de la Estratigrafía del Valle de Santa María, Provincia de Catamarca, Argentina. In: CONGRESO LATINOAMERICANO DE GEOLOGÍA, 5, 1982. Resúmenes, Buenos Aires, p. 155-172.

Cabrera, A.L. 1976. Territorios Fitogeográficos de la República Argentina. In: L.R. Parodi (ed.) Enciclopedia Argentina de Agricultura y Jardinería, Editorial ACMÉ, Tomo 1, 85 p.

Caccavari, M.A. 1979. Granos de polen de Nictagináceas Argentinas. Revista del Museo Argentino de Ciencias Naturales Bernardino Rivadavia, 5(10):211-234.

Cione, A.C. \& Báez, A.M. 2007. Peces continentales y anfibios cenozoicos de Argentina: los últimos cincuenta años. In: S. Archangelsky; T. Sánchez \& E.P. Tonni (eds.) Ameghiniana 50, Publicación Especial, 11:173-191.

Clarke, R.T. \& Frederiksen, N.O. 1968. Some new sporomorphs from the Upper Tertiary of Nigeria. Grana Palynologica, 8:210224. doi:10.1080/00173136809427466

Couper, R.A. 1960. New Zealand Mesozoic and Cainozoic plant microfossils. New Zealand Geological Survey Paleontological Bulletin, 32:1-87.

Covas, G. 1941. Las Amarantáceas bonariensis. Darwiniana, 5:329-368.

Cronquist, A. 1981. An Integrated System of Classification of Flowering Plants. New York, Columbia University Press, 1262 p.

Elsik, W.C. 1969. Late Neogene palynomorph diagrams, Northern Gulf of Mexico. Transactions-Gulf Coast Association of Geological Societes, 19:509-528.

Ezcurra, C. 2002. El género Justicia (Acanthaceae) en Sudamérica Austral. Annals of the Missouri Botanical Garden, 89: 225-280.

Giusti, L. 1987. Chenopodiaceae. In: N.S. Troncoso \& N.M. Bacigalupo (eds.) Fl. 2 Entre Ríos, Colecciones de Ciencias Naturales del Instituto Nacional de Tecnología Agropecuaria, 6(3a):137-159.

Germeraad, J.H.; Hopping, C.A. \& Muller, J. 1968. Palynology of Tertiary sediments from tropical areas. Review of Palaeobotany and Palynology, 6:189-348. doi:10.1016/0034-6667(68)90051-1

Graham, A. 1976. Studies in Neotropical Paleobotany. 2. The Miocene communities of Veracruz, México. Annals of Missouri Botanical Garden, 63:787-842.

Graham, A. 1988. Studies in Neotropical Paleobotany. 6. The Lower Miocene communities of Panama. The Cucaracha Formation. Annals of Missouri Botanical Garden, 75:1467-1479.

Guerstein, G.R.; Guler, M.V. \& Casadío, S. 2001. Palynostratigraphy and palaeoenvironments across the Oligocene-Miocene boundary within the Centinela Formation, Southwestern Argentina. The Palynology and Micropaleontology of Boundaries, 230:325-343.

Guler, M.V.; Guerstein, G.R. \& Quattrocchio, M.E. 2001. Palinología del Neógeno de la Perforación Cx-1 Cuenca del Colorado, Argentina. Revista Española de Micropaleontología, 33:183-204.

Hauman, L.L. 1924. Notes floristiques. Anales del Museo Nacional de Historia Natural de Buenos Aires, 32:439-475.

Ibáñez, L. 2001. Análisis paleoambiental de la Formación Chiquimil en el valle de Santa María, Catamarca, Tucumán, Argentina. Universidad Nacional de Tucumán, Tesis Doctoral, 199 p.

Jansonius, J. \& Hills, L.V. 1976. Genera file of fossil spores. Department of Geology, University of Calgary, Special publication, Reference Card No. 3-431 cards.

Jarzen, D.M. \& Dettmann, M.E. 1989. Taxonomic revision of 
Tricolpites reticulatus Cookson ex Couper, 1953 with notes on the biogeography of Gunnera L. Pollen et Spores, 31:97-112.

Jarzen, D.M. \& Dilcher, D.L. 2006. Middle Eocene terrestrial palynomorphs from the Dolime Mineral and Gulf Hammock Quarries, Florida, U.S.A. Palynology, 30:90-110.

Kiesling, R. 1978. El género Trichocereus (Cactaceae) 1: Las especies de la Rep. Argentina. Darwiniana, 21:263-330.

Kiesling, R. 1998. Notas sobre cactáceas 1. Candollea, 53:471-476.

Kleinert, K. \& Strecker, M.R. 2001. Climate change in response to orographic barrier uplift: paleosol and stable isotope evidence from the late Neogene Santa Maria basin, northwestern Argentina. Geological Society of America, 6:728-242.

Krutzsch, W. 1966. Zur Kenntnis der präquartären peripotaten Pollenformen. Geologie, 55: 16-71.

Latorre, C.; Quade, J. \& McIntosh, W.C. 1997. The expansion of $\mathrm{C} 4$ grasses and global cange in the table Miocene: stable isotope evidence from the Ameritas. Earth and Planetary Science Letters, 146:83-96. doi:10.1130/0016-7606

Leuenberger, B.E. 1976. Die Pollenmorphologie der Cactaceae. Dissertationes Botanicae, 31:1-321.

Lorente, M.A. 1986. Palynology and palynofacies of the Upper Tertiary in Venezuela. Dissertationes Botanicae, 99:1-222.

Lucena, I.D. 1993. Nyctaginaceae. Flora del Valle de Lerma. Aportes Botánicos de Salta, Série Flora, 1(24):1-22.

Markgraf, V. \& D'Antoni, H.L. 1978. Pollen flora of Argentina. Modern spore and pollen types of Pteridophyta, Gymnospermae, and Angiospermae. Tucson, University of Arizona Press, 208 p.

Martin, H.A. 1973. The palynology of some Tertiary and Pleistocene deposits, Lanchan River Valley, New South Wales. Australian Journal of Botany, Supplentary Series, 6:1-57.

Martinez, L.C.A. 2010. Prosopisinoxylon anciborae nov. gen. et sp. (Leguminosae, Mimosoideae) from the Late Miocene Chiquimil Formation (Santa María Group), Catamarca, Argentina. Review of Paleobotany and Palynology, 158(3/4):262271. doi:10.1016/j.revpalbo.2009.09.006

Mathur, Y.K. 1966. On the Microflora in the Supra-Trappeans of Western Kutch, India. Quaterly Journal of the Geological Mining and Metalurgical Society of India, 38(1):33-51.

Mautino, L.R. 2007. Chlorophyta de los Valles Calchaquíes (Mioceno Medio y Superior), Argentina. Revista Española de Micropaleontología, 39(1/2):81-102.

Mautino, L.R. 2010. Palinofloras de las Formaciones San José y Chiquimil (Mioceno Medio y Superior), Noroeste de Argentina. Universidad Nacional del Nordeste, Tesis Doctoral, 444 p.

Mautino, L.R. \& Anzótegui, L.M. 1998. Palinología de la Formación Chiquimil (Mioceno Superior) Localidad Vallecito, Provincia de Catamarca. Parte 1: Especies Nuevas. Ameghiniana, 35(2):227-233.

Mautino, L.R. \& Anzótegui, L.M. 2000. Esporas del Mioceno y retrabajadas del Mesozoico en la Formación Chiquimil, Vallecito, Provincia de Catamarca. Ameghiniana, 37(1):13-22.

Mautino, L.R. \& Anzótegui, L.M. 2002a. Palinología de la Formación Chiquimil (Mioceno Superior), Vallecito, Provincia de Catamarca. Parte 2. Polen. Ameghiniana, 39(3):257-270.

Mautino, L.R. \& Anzótegui, L.M. 2002b. Palinología de la Formación Chiquimil (Mioceno Superior) Vallecito, Provincia de Catamarca. Parte 3. Polen. Ameghiniana, 39(3):271-284.

Mautino, L.R.; Anzótegui, L.M. \& Herbst, R. 1997. Análisis palinológico de la localidad Nacimientos de Abajo, en la sierra de Hualfin, Departamento Belén, Catamarca, Argentina. Geociencias, 2:121-127.

Mautino, L.R.; Cuadrado, G. \& Anzótegui L. 2004. Novedades taxonómicas, diversidad y significado evolutivo del polen de
Malvaceae en el Terciario de Argentina. Revista Española de Micropaleontología, 36:467-483.

Mildenhall, D.C. \& Pocknall, D.T. 1989. Miocene-Pleistocene Spores and Pollen from Central Otago, South Island, New Zealand. New Zealand Geological Survey, Paleontological Bulletin, 59:1-128.

Morton, L.S. 2004. Taxonomia y paleoecología de la malacofauna (Bivalvia-Gastropoda) dulceacuícola del Neógeno, en los Valles de Santa María y del Cajón, Catamarca y Tucumán, Argentina. Universidad Nacional del Nordeste, Tesis doctoral, 134 p.

Muller, J.; Di Giacomo E. \& van Erve, A.W. 1987. A palynological zonation for the Cretaceous, Tertiary, and Quaternary of Northen South America. American Association of Stratigraphic Palynologists, Contribution Series, 19:1-71.

Ottone, E.G.; Barreda, V.D. \& Pérez, D.J. 1998. Basin evolution as reflected by Miocene palynomorphs from the Chinches Formation, Frontal Cordillera $\left(32^{\circ} \mathrm{S}\right)$, San Juan Province, Argentina. Revista Española de Micropaleontología, 30(3):35-47.

Palamarczuk, S. \& Barreda, V.D. 2000. Palinología del Paleógeno tardío-Neógeno temprano, pozo Aries x-1, plataforma continental argentina, Tierra del Fuego. Ameghiniana, 37(2):221-234.

Pire, S.M.; Anzótegui, L.M. \& Cuadrado, G.A. 1998. Flora Polínica del Nordeste Argentino 1. EUDENE. UNNE. 143p.

Pire, S.M.; Anzótegui, L.M. \& Cuadrado, G.A. 2006. Flora Polínica del Nordeste Argentino. Corrientes, EUDENE/UNNE, 172 p.

Regali, M.S.P.; Uesugui, N. \& Santos, A.S. 1974. Palinologia dos sedimentos Meso-Cenozóicos do Brasil - II. Boletim Técnico da Petrobras, 17:263-301.

Scholtz, A. 1985. The palynology of the upper lacustrine sediments of the Arnot Pipe, Banke Namaqualand. Annals of the South African Museum, 95:1-109.

Sohmer, S.H. 1976. Herbstia, a new genus in the Amaranthaceae. Brittonia, 28:448-452.

Stover, L.E. \& Partridge, A.D. 1973. Tertiary and Late Cretaceous spores and pollen from the Gippsland Basin, Southeastern Australia. Proceedings of the Royal Society of Victoria, 85:237-286.

Thomson, P.W. \& Pflug, H. 1953. Pollen und sporen des mitteleuropäischen Tertiärs. Palaeontographica, 94:1-138.

Toursarkissian, M. 1975. Las Nictagináceas Argentinas. Revista Museo Argentino de Ciencias Naturales Bernardino Rivadavia, 5:27-83.

Truswell, E.M.; Sluiter, I.R. \& Harris, W.K. 1985. Palynology of the Oligocene-Miocene sequence in the Oakvale-1 core hole, Western Murray Basin, South Australia. Journal of Australian Geology and Geophysics, 9:267-295.

Van der Hammen, T. 1956. Description of some genera and species of fossil pollen and spores. Boletin Geológico, 4:63-101.

Yamamoto, T.I. 1995. Palinologia das bacias do sudeste (Bacias de Taubaté, São Paulo e Resende). Análise Bioestratigráfica integrada e interpretação paleoambiental. Instituto de Geociências e Ciências Exatas, Universidade Estadual Paulista, Dissertação de Mestrado, 217 p.

Zamaloa, M. del C. 2000. Palinoflora y ambiente en el Terciario del nordeste de Tierra del Fuego, Argentina. Revista del Museo Argentino Ciencias Naturales Bernardino Rivadavia, 2:43-51.

Zetter, R.C.C.; Hofmann, I.D.; Durango de Cabrera, J.; Vergel, M.M. \& Vervoorst, F. 1999. A rich Middle Eocene microflora at Arroyo de los Mineros, near Cañadón Beta, NE Tierra del Fuego Province, Argentina. Abhandlungen der Geologischen Bundesanstalt, 56:436-460.

Zuloaga, F.O. \& Morrone, O. 1999. Catálogo de las plantas vasculares de la República Argentina 1 y 2. Saint Louis, Missouri Botanical Garden Press, 1270 p.

Received in May, 2011; accepted in October, 2011. 